\title{
La tombe monumentale de Grotte Scalina (Viterbe) Campagne de fouilles 2017
}

Vincent Jolivet et Edwige Lovergne

\section{(2) OpenEdition \\ Journals}

Édition électronique

URL : http://journals.openedition.org/cefr/1987

DOI : $10.4000 /$ cefr. 1987

ISSN : 2282-5703

\section{Éditeur}

École française de Rome

\section{Référence électronique}

Vincent Jolivet et Edwige Lovergne, « La tombe monumentale de Grotte Scalina (Viterbe)

Campagne de fouilles 2017 », Chronique des activités archéologiques de l'École française de Rome [En

ligne], Italie centrale, mis en ligne le 30 juillet 2018, consulté le 01 mai 2019. URL : http://

journals.openedition.org/cefr/1987 ; DOI : 10.4000/cefr.1987

Ce document a été généré automatiquement le 1 mai 2019.

(c) École française de Rome 


\title{
La tombe monumentale de Grotte Scalina (Viterbe) Campagne de fouilles 2017
}

\author{
Vincent Jolivet et Edwige Lovergne
}

\section{NOTE DE L'ÉDITEUR}

Ont participé aux travaux de la campagne 2017 : Elisa Abbondanzieri (Roma, Università La Sapienza), Alice Baud (université de Paris I), Sophie Brones (Paris), Virginie Boutet (Paris), Thomas Broise (Aix-en-Provence), Anaïs Daumont-Marx (Paris), Manuel de Souza (université de Saint-Étienne), Saddam Douadi (Chlef, Algeria), Pauline Ducret (Paris, École normale supérieure), Hélène Dufresne (université de Paris I), Baptiste Duvert (université de Tours), Audrey Gouy (Paris, École Pratique des Hautes Études), Serenella Isidori (Università della Tuscia, Viterbo), Julie Labregère (université de Tours), Élisa Mathieu (Paris), Lucie Motta (université de Lyon), Pascal Neaud (INRAP, Reims), Mathieu Niveleau (Paris), Luca Pesante (Roma), François-Xavier Romanacce (université de Paris Sorbonne - Abou Dhabi), Gabi Seiwerth (Wien), Frédéric Tollinchi (Marseille) e Emma Vallet (université de Lyon).

1 La septième campagne de fouilles sur le site de la tombe monumentale de Grotte Scalina, placée sous le régime de la concession octroyée par le Ministero dei Beni e delle Attività Culturali e del Turismo à l'École normale supérieure de Paris, s'est déroulée du 3 juillet au 5 août 2017 sous la direction scientifique de Vincent Jolivet et sous la direction opérationnelle d'Edwige Lovergne, en étroite collaboration avec la Soprintendenza Archeologia, Belle Arti e Paesaggio per l'area metropolitana di Roma, la provincia di Viterbo e l'Etruria meridionale (inspectrice : Dott.ssa Valeria D'Atri) ; le plan de sécurité a été rédigé par l'architecte Alessandro Bertollini, les travaux de chantier réalisés par l'entreprise Enzo Nicolamme de Monterazzano. Anaïs Daumont Marx et Pascal Neaud ont exercé la responsabilité de différents secteurs du chantier. Le financement a été assuré 
par l'UMR 8546 (AOrOc) du CNRS, le Département des Sciences de l'Antiquité de l'École normale supérieure de Paris, l'ANR CAECINA, le Labex TransferS (programme Investissements d'avenir ANR-10-IDEX-000102 PSL* et ANR-10-LABX-0099) et la Fondazione Carivit de Viterbe. Maria et Enzo Pepponi, propriétaires du site, ainsi que Felice Orlandini, inspecteur honoraire des Biens Culturels, ont largement contribué, de multiples manières, à la réalisation de l'ensemble de l'opération.

2 Les relevés ont été effectués par Filippo Bozzo (Università della Tuscia, Viterbo), la couverture photographique de la campagne de fouille par Gabi Seiwerth (Vienne, Autriche), les vues par drone de l'ensemble du site et de la vallée de la Leia par Benjamin Houal (Paris). Les recherches portant sur l'hypogée principal ont été réalisées par l'entreprise UNIGEO, sous la direction de Daniele D'Ottavio, et par l'Associazione Roma Sotterranea, sous la direction de Marco Placidi.

3 Nos travaux ont porté principalement cette année sur trois secteurs: le complexe funéraire monumental, le champ qui le surmonte, et le petit oppidum dit de l'Isolotto.

4 L'achèvement de la tranchée ouverte l'an dernier sur toute la hauteur de la colline, en axe avec le dromos de la tombe, a permis d'établir qu'il n'a jamais existé - du moins dans ce secteur - de dispositif d'accès direct à la tombe à partir de la route antique qui passait en contrebas, dont aucune trace n'a été découverte - probablement parce qu'elle a été utilisée sans solution de continuité, par la suite, de l'époque médiévale à nos jours. Il est donc très probable que l'on accédait à la tombe au niveau du départ de son dromos par une voie secondaire créée à mi-pente de la colline, dont la présence est indirectement attestée par celle des tombes archaïques découvertes en 2013 au sud-ouest du monument. Le tracé de cette voie semble avoir été repris à l'époque moderne, comme en témoigne le mur de soutènement formé de gros blocs grossièrement équarris découvert l'an dernier au sommet de la tranchée. Le dégagement des blocs provenant de l'effondrement de la tombe, réalisé pour adapter celle-ci à sa nouvelle fonction rituelle, et l'aménagement de la nouvelle voie peuvent être datés, sur la base du mobilier recueilli, autour du milieu du $\mathrm{XVI}^{\mathrm{e}}$ siècle. Les travaux réalisés autour de la tombe ont par ailleurs permis la découverte d'une monnaie frappée au cours de l'année jubilaire 1600, sous le pontificat de Clément VIII (fig. 1), qui présente le double intérêt de confirmer le lien du site avec le Jubilé romain et de permettre d'attribuer à une période antérieure à cette date la refonctionnalisation de la tombe à des fins rituelles. Elle présente sur une face le blason du souverain pontife, sur l'autre la Porta Santa. Enfin, nous avons achevé cette année la consolidation du dromos de la tombe, dont les fragiles parois s'étaient effondrées par endroits. 


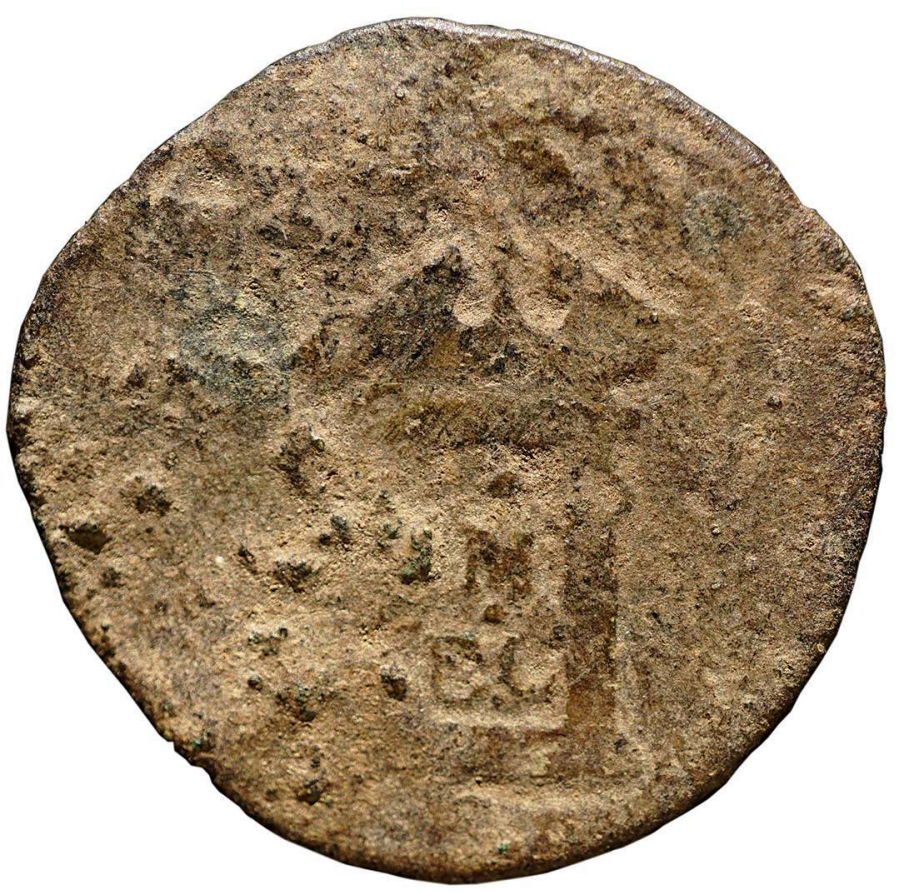

G. Seiwerth.

Le prolongement de l'enquête géophysique réalisée l'an dernier, qui avait mis en évidence la présence probable d'un puits rectangulaire creusé au centre de l'hypogée principal, a entraîné un certain nombre d'opérations visant à localiser la chambre originaire de la tombe, dont on peut supposer la présence sur la base de différents indices concordants : contraste entre le soin apporté à l'architecture extérieure et la taille très négligée des deux tombes ; qualité médiocre des sarcophages ; rareté et pauvreté des inscriptions ; mobilier recueilli entièrement postérieur, de deux générations environ, à l'utilisation de la salle de banquet funéraire, autour de 320 av. J.-C. Au centre de l'hypogée principal, une perforation profonde de $15 \mathrm{~m}$, réalisée à l'emplacement du puits présumé, a permis de conclure à l'absence de tout vide sous-jacent, et d'établir que le terrain, très meuble, ne se prêtait pas à la création d'une autre chambre funéraire : si le puits mis en évidence par la prospection géophysique a bien existé, il doit donc être considéré comme une tentative réalisée par les créateurs de la tombe d'atteindre, sans succès, des couches de tuf compact. D'autres enquêtes réalisées dans ce même hypogée - déplacement d'un sarcophage, sondage dans une paroi - n'ont abouti à aucun résultat décisif, même si elles semblent indiquer la présence d'un espace vide, en contact avec la surface, au nord-ouest de l'hypogée principal. On peut donc supposer que les créateurs de la tombe, après avoir constaté la faible qualité du terrain dans la partie inférieure de la colline, ont décidé de creuser l'hypogée au-dessus, à l'intérieur de la couche de tuf compacte dans laquelle a été réalisée la façade. S'il en est bien ainsi, l'accès à la chambre funéraire originaire du complexe pourrait avoir été aménagé à partir du plateau qui domine la tombe. Cette nouvelle hypothèse devrait faire l'objet de vérifications ultérieures. 
6 Sur ce même plateau, les recherches précédentes, ainsi que différentes photographies aériennes, de la dernière guerre mondiale à nos jours, avaient permis de localiser un petit (ca. $250 \mathrm{~m}^{2}$ ) site médiéval datable, sur la base de la fouille d'un tronçon de son enceinte, en 2013, des XII ${ }^{\mathrm{e}}$-XIII ${ }^{\mathrm{e}}$ siècles, et qui semblait délimité, vers l'ouest, le nord et l'est, par un large fossé. La tranchée de 2,40 × 18,60 m ouverte perpendiculairement à ce dernier (fig. 2 ), pour une profondeur maximale de $2 \mathrm{~m}$, a permis d'établir qu'il s'agissait en fait d'une ligne de front de carrière, au-dessus de laquelle avait été construit le mur d'enceinte du site, entièrement détruit, avec l'ensemble des constructions et des niveaux archéologiques qui s'y rapportaient, par les importants travaux de bonification agricole réalisés dans cette zone. Cette carrière présente cependant deux types différents de traces d'extraction: le plus ancien, destiné à extraire de gros blocs rectangulaires, peut être daté, également sur la base d'un fragment de céramique, de la fin du $\mathrm{IV}^{\mathrm{e}}$ siècle, et donc de l'époque de création de la tombe ; le plus récent, correspondant à de petits blocs carrés, se rapporte au site médiéval, dont la datation a pu être confirmée par le riche mobilier recueilli cette année.

Fig. 2 - Plan de la tranchée : à droite, la carrière ; à gauche, l'emplacement du site arasé.

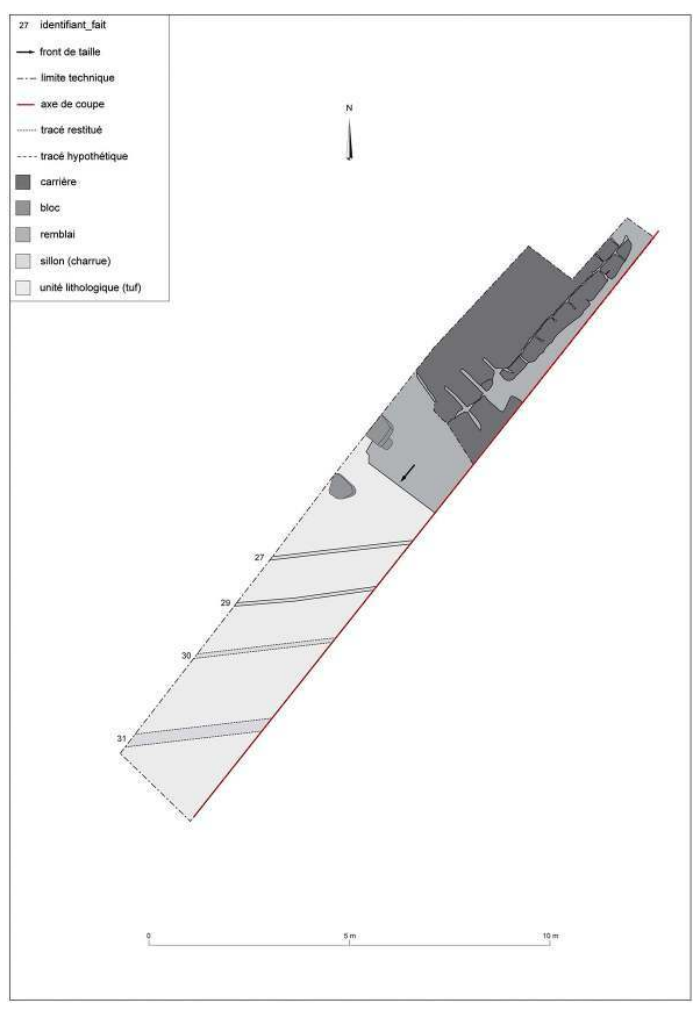

P. Neaud.

7 Sur le petit oppidum de l'Isolotto, une centaine de mètres au sud-est de la tombe, la fouille complète des structures conservées (fig. 3), qui occupent une superficie d'environ $100 \mathrm{~m}^{2}$, a permis d'établir définitivement qu'elles appartenaient à un fortin du début de l'époque hellénistique, construit en grand appareil de tuf, et probablement doté d'une tour de guet, qui faisait partie du système défensif de Musarna en assurant, vers le nord, le contrôle de l'important axe de circulation reliant Sorrina-Viterbe à Tuscania. Différents éléments, dont un bassin soigneusement construit en fragments de tegulae et revêtu d' opus signinum, confirment la réoccupation du site au début de l'époque impériale, que la 
fouille de la citerne appartenant au complexe, contenant un abondant mobilier d'époque claudienne, avait déjà permis d'établir: il semble s'agir d'une réutilisation liée à une modeste activité agricole. Nous n'avons relevé, en revanche, aucune trace d'occupation du site au Moyen-Âge.

Fig. 3 - Orthomosaïque de l'oppidum de l'Isolotto.

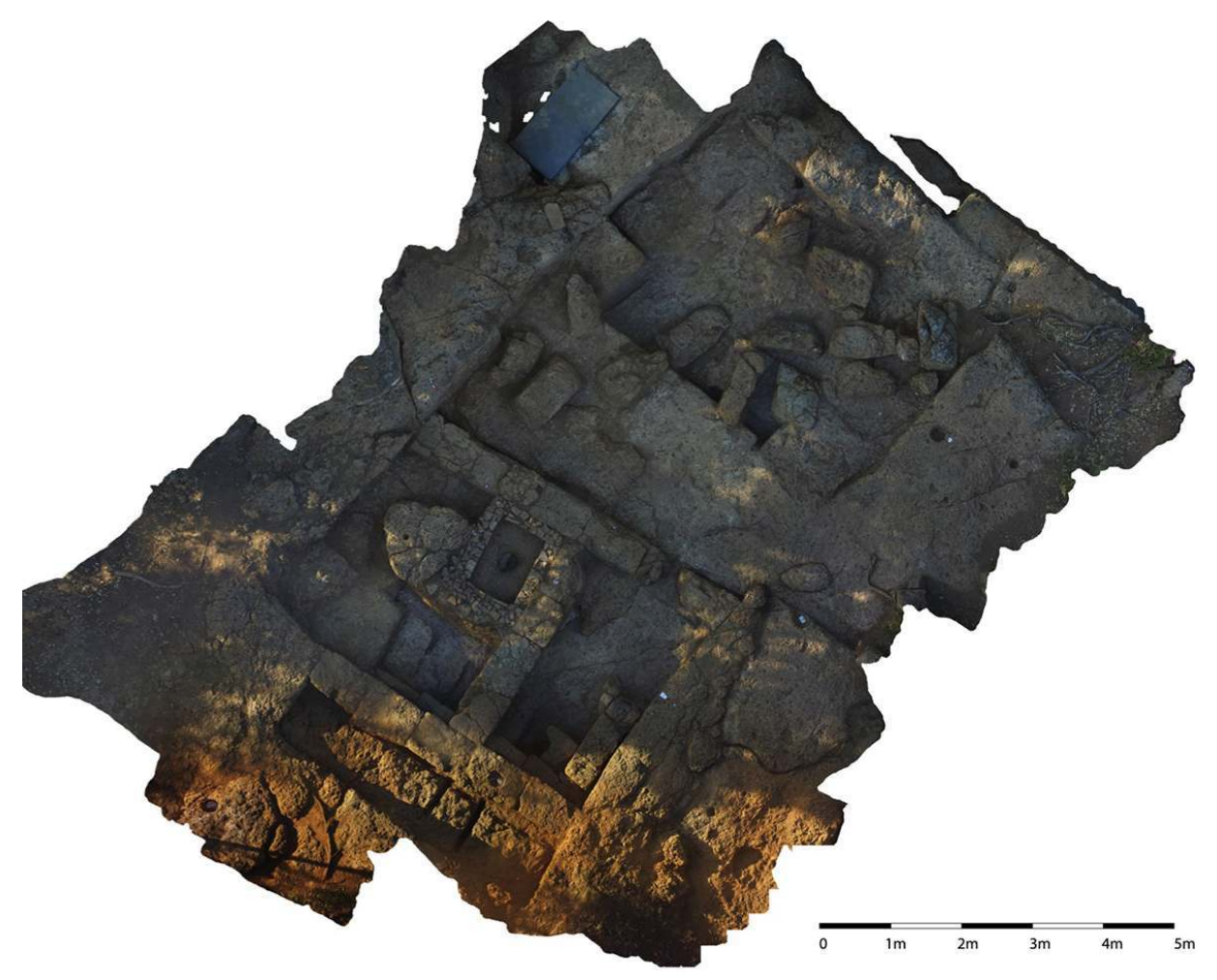

B. Houal.

Deux autres opérations, sans lien avec la tombe, ont également été menées cette année. À l'ouest de la ferme Pepponi, les travaux agricoles avaient mis au jour un fragment d'architrave de nenfro portant trois lettres d'une inscription étrusque de grandes dimensions. La fouille ouverte dans ce secteur, préparée par une prospection géophysique, a permis d'établir que ce bloc avait été utilisé pour revêtir la paroi d'un four à chaux de forme ovale, qu'aucun élément ne permet actuellement de dater. Le démontage systématique de ce revêtement, fait de blocs de tuf et de nenfro partiellement calcinés, n'a pas permis la découverte d'autres blocs inscrits appartenant au même monument.

Enfin, l'hypogée hellénistique connu sous le nom de Grotta delle Statue, au nord-est du château de Respampani, a fait l'objet d'un nettoyage complet, de manière à en réaliser le relevé photogrammétrique et une nouvelle couverture photographique. Le sondage ouvert au-dessus de la tombe, où le banc de roche est scellé par une faible couche de terre, n'a pas permis de mettre au jour les traces d'un monument funéraire construit.

10 Si la fouille de la tombe hellénistique de Grotte Scalina peut donc être considérée, à ce stade, comme achevée, les recherches se poursuivront pour tenter de localiser la première chambre funéraire du complexe - si celle-ci a bien existé. 


\section{BIBLIOGRAPHIE}

Jolivet - Motta 2009 = V. Jolivet, L. Motta, Musarna: Macchia del Conte Vecchia, REE, dans SE, 75, 2009, p. 252-253.

Jolivet - Lovergne $2014=$ V. Jolivet, E. Lovergne, La tomba rupestre monumentale di Grotte Scalina (VT), dans L. Mercuri, R. Zaccagnini (dir.), Atti del convegno Etruria in Progress. La ricerca archeologica in Etruria meridionale, Rome, 2014, p. 165-170.

Jolivet - Lovergne $2015=$ V. Jolivet, E. Lovergne, Architecture palatiale macédonienne, architecture funéraire étrusque : la tombe monumentale de Grotte Scalina (Viterbe), dans Actes du colloque de Merida, Madrid, 2015, p. 47-50.

Jolivet - Lovergne $2016 \mathrm{a}=\mathrm{V}$. Jolivet, E. Lovergne, La tombe rupestre monumentale de Grotte Scalina (Étrurie méridionale), dans RA, 2016, p. 151-162.

Jolivet - Lovergne 2016b = V. Jolivet, E. Lovergne, Etruscan puzzle: the monumental tomb of Grotte Scalina, dans World Archaeology, 80, 2016, p. 26-30.

Jolivet $2016=$ V. Jolivet, Macedonia and Etruria at the beginning of the Hellenistic period: a direct link,

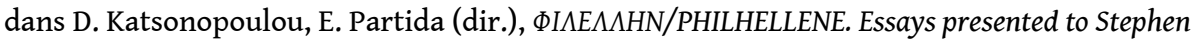

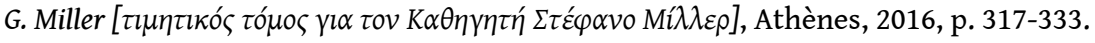

Jolivet - Lovergne 2017 = V. Jolivet, E. Lovergne, The monumental tomb of Grotte Scalina: solved and unsolved questions, dans Etruscan News, 18, 2017, p. 10.

\section{INDEX}

institutions Soprintendenza Archeologia, Belle Arti e Paesaggio per l'area metropolitana di Roma, la provincia di Viterbo e l'Etruria meridionale, AOROC (UMR 8546 CNRS), École normale supérieure (Paris)

\section{AUTEURS}

\section{VINCENT JOLIVET}

CNRS, AOROC (UMR 8546 CNRS-ENS) - vincent.jolivet@ens.fr

\section{EDWIGE LOVERGNE}

UMR 7041 (ArScAn) - edwigelovergne@hotmail.com 